\title{
Achievable Localization Accuracy of the Positioning Reference Signal of 3GPP LTE
}

\author{
José A. del Peral-Rosado, José A. López-Salcedo \\ and Gonzalo Seco-Granados \\ Universitat Autònoma de Barcelona (UAB) \\ Bellaterra, Spain \\ Email: \{JoseAntonio.DelPeral, Jose.Salcedo, \\ Gonzalo.Seco\}@uab.cat
}

\author{
Francesca Zanier \\ and Massimo Crisci \\ European Space Agency (ESA) \\ Noordwijk, The Netherlands \\ Email: \{Francesca.Zanier, Massimo.Crisci\}@esa.int
}

\begin{abstract}
Robustness of nominal Global Navigation Satellite Systems (GNSS) performance can be enhanced by means of complimentary systems, such as the Long Term Evolution (LTE). Particularly, the LTE standard specifies a dedicated downlink signal for positioning purposes, i.e. the positioning reference signal (PRS). This paper presents the achievable localization accuracy of the PRS signal for different interference LTE scenarios by means of the Crámer-Rao bound (CRB) for time delay estimation, in order to assess the LTE positioning capabilities.
\end{abstract}

\section{INTRODUCTION}

The introduction of mass-market Global Navigation Satellite System (GNSS) receivers in mobile phones and portable devices has given rise a myriad of possible working conditions. Mobile terminals can be operated either outdoors or indoors, in sparsely populated areas or in deep urban scenarios, and thus robustness is critical for localization purposes in those conditions. Certainly, huge efforts have been devoted to the enhancement of GNSS receivers in challenging environments, but they find serious difficulties to achieve the demanded performance. It is for this reason that complementary systems are usually proposed to assist the operation of GNSS systems. Traditionally, assistance data provided by an external source, such as a cellular network, or inertial navigation systems (INS) has been introduced in harsh environments. Moreover, other alternatives, such as the hybridization with signals of opportunity (SoO) or backup systems, have recently been considered, too.

A notable example of signal of opportunity or backup system is the Long Term Evolution (LTE) specified by the $3^{\text {rd }}$ Generation Partnership Project (3GPP) consortium [1]. This technology, which has received significant interest from mobile network providers, is expected to be highly deployed with a significant coverage. More importantly, LTE incorporates positioning capabilities to satisfy two main drivers: legal mandates for location identification of emergency calls (e.g. E911 in US or E112 in Europe), and commercial applications or location-based services (LBS), such as navigation, advertising or social media. LTE adopts assisted-GNSS (AGNSS), as a primary positioning method, and the enhanced Cell-ID (e-CID) and the Observed Time Difference of Arrival (OTDoA), as complimentary methods. Our interest is focused on OTDoA due to the use of a dedicated downlink signal for positioning, i.e. the positioning reference signal (PRS), which is a multicarrier Orthogonal Frequency Division Multiplexing (OFDM) signal. Multicarrier signals are well-known in wireless communications because of their flexibility, which offers spectral efficiency, and robustness against frequency-selective fading introduced by multipath, among other advantages with respect to traditional single-carrier signals.

The aim of this paper is to find the achievable localization accuracy of the LTE positioning reference signal from the maximum likelihood point of view. Following the preliminary analysis on the LTE signal structure shown in [2], the CrámerRao bound (CRB) for time delay estimation is also introduced to assess the positioning capabilities in additive white Gaussian noise (AWGN) channel, presenting a feasible estimator. Then, the interference case is applied to the LTE scenario simulations and final position errors are shown in terms of the CRB for OTDoA localization. Finally, we draw the conclusions.

\section{LONG TERM EVOLUTION (LTE)}

The Long Term Evolution (LTE) moves towards the fourth generation $(4 \mathrm{G})$ of mobile communications. Mostly of its standard, which is driven by the 3GPP, has been inherited from the Universal Mobile Telecommunication System (UMTS) in order to maintain backward compatibility. The main new features introduced are the downlink Orthogonal FrequencyDivision Multiple Access (OFDMA) and the Multiple Input Multiple Output (MIMO) data transmission. The signal bandwidth is scalable from $1.4 \mathrm{MHz}$ to $20 \mathrm{MHz}$ with a symbol period $T_{s}$ of $66.67 \mu \mathrm{s}$, which corresponds to a subcarrier spacing $F_{s c}$ of $15 \mathrm{kHz}$.

According to the LTE specification [3], the downlink positioning procedure, defined by the OTDoA method, uses the difference in the arrival times of downlink radio signals from multiple base stations (i.e. eNodeBs) to compute the user position. The method relies on a network-based strategy because the eNodeB locations are not provided to the user. First, the user equipment (UE) requests assistance information to proceed with the timing measurements. Then, the LTE Positioning Protocol (LLP) transfers the UE measurements to 


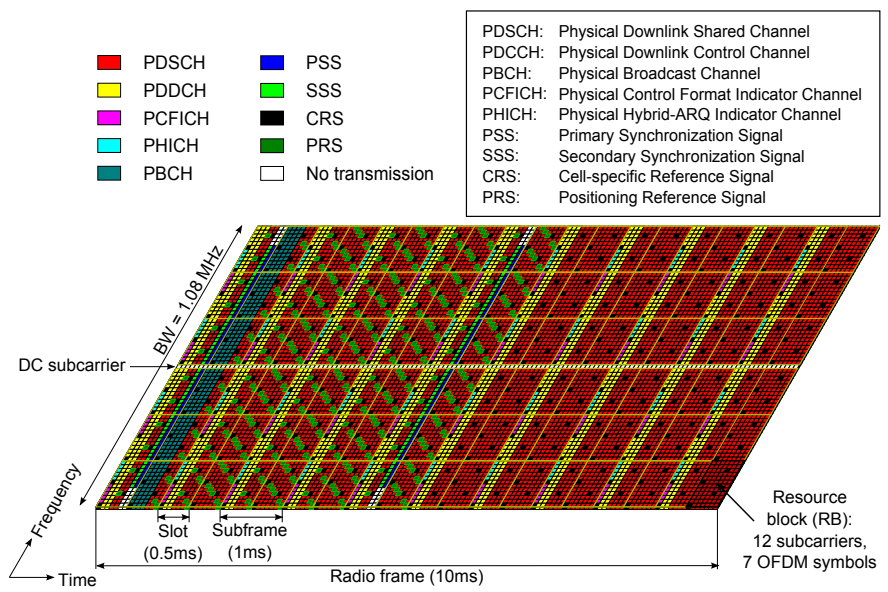

Fig. 1. Time-frequency grid of the LTE signals for $1.4 \mathrm{MHz}$ bandwidth, FDD structure and normal cyclic prefix $(\mathrm{CP})$.

the location server, E-SMLC (Enhanced Serving Mobile Location Center). Based on the UE measurements, the E-SMLC estimates the UE position using a trilateration technique, and this position information is finally sent back to the user.

The LTE standard [4] specifies a set of downlink signals based on an OFDM modulation with different timefrequency distribution, whose basic structure is shown in Fig. 1. Downlink synchronization and reference signals are completely known (like the pilot signals in GNSS), thus they are suitable for ranging purposes, or even for SoO applications, such as the primary and secondary synchronization signal (i.e. PSS and SSS), as well as the cell-specific reference signal (CRS), that do not require any assistance data. However, LTE follows the typical frequency reuse factor of a cellular network, which is equal to one. Thus, the received serving cell signal interferes with the received neighbour cell signals producing inter-cell interference, and resulting in the nearfar effect. In order to obtain proper ranging measurements of the neighbour cells, the LTE standard in Release 9 specifies a positioning reference signal (PRS) that is especially dedicated for positioning purposes and mitigates the near-far effect, due to a higher frequency reuse factor (i.e. of six), by shifting one subcarrier position the frequency pilot allocation transmitted by each base station. The main parameters for PRS configuration are shown in Table I. The PRS signal is scattered in time and frequency in the so-called positioning occasion, which allocates consecutive positioning subframes with a certain periodicity. The sophistication of this signal is even higher when the network mutes the PRS transmissions of certain base stations (i.e. PRS muting), in order to further reduce the inter-cell interference.

\section{TIME DELAY ESTIMATION}

The performance of the time delay estimation (TDE) can be assessed by establishing an accuracy limit. Thus, a lower bound on timing estimation can help us to analyse the time synchronization algorithm implemented. For this analysis, the cyclic prefix $(\mathrm{CP})$ is already removed in a previous stage.
TABLE I

MAIN PARAMETERS OF THE PRS SIGNAL.

\begin{tabular}{ll}
\hline PRS bandwidth & $1.4,3,5,10,15$ and $20 \mathrm{MHz}$ \\
PRS periodicity & $160,320,640$ or $1280 \mathrm{~ms}$ \\
Consecutive subframes & $1,2,4$, or 6 \\
PRS muting information ${ }^{1}$ & 2, 4, 8, 16 bits \\
PRS pattern & 6-reuse in frequency \\
PRS sequence & Length-31 Gold sequence
\end{tabular}

${ }^{1}$ Number of positioning occasion configured for PRS muting (i.e. bit equal to 0 when PRS is muted).

\section{A. Crámer-Rao bound (CRB)}

The Crámer-Rao bound (CRB) is a well-known lower bound that describes the maximum achievable accuracy of any unbiased estimator in the moderate- to high-SNR region. Since the time delay is estimated with pilot sequences, the CRB can be analytically computed.

Let us define the OFDM baseband signal format for one symbol used in the LTE downlink (without CP) as

$$
x[n]=\sqrt{\frac{2 \cdot C}{N_{c}}} \sum_{k \in \mathcal{N}_{a}} p_{k} \cdot d_{k} \cdot \exp \left(j \frac{2 \pi n k}{N_{c}}\right),
$$

where $C$ is defined as the power of the band-pass signal, $N_{c}$ is the number of subcarriers (excluding unused DC subcarrier), $\mathcal{N}_{a}$ is the subset of active pilot subcarriers $N_{a}$, which must satisfy $N_{a} \leq N_{c}, d_{k}$ are the symbols, and $p_{k}^{2}$ is the relative power weight of subcarrier $k$, which is constrained by $\sum_{k} p_{k}^{2}=N_{c}$ to give the nominal signal power $C$. This notation allows the flexible design of OFDM multicarrier signal optimizing the power and spectra distribution. It also has to be noticed that the OFDM symbol duration $T_{s}$ is inverse to the subcarrier spacing $F_{s c}$, i.e. $T_{s}=1 / F_{s c}$. In addition, the OFDM signal generation can be efficiently implemented with the inverse fast Fourier transform (IFFT) of the pilot symbols vector $\mathbf{d}$.

Since the OFDM signal described in (1) is completely known, the Cramér-Rao Bound (CRB) expression for time delay estimation, $\hat{\tau}$, applied to the LTE signal formats can be derived from the general definition given by Kay [5],

$$
\operatorname{var}(\hat{\tau}) \geq \operatorname{CRB}(\tau)=\frac{1}{\frac{E_{s}}{N_{0} / 2} \cdot \bar{F}^{2}},
$$

where $E_{s}=C \cdot T_{s}$ and $\mathrm{SNR}=\left(C / N_{0}\right) / B$, being $C / N_{0}$ the carrier-to-noise-density ratio and $B$ the bandwidth of the signal. The mean square bandwidth (MSB) or Gabor bandwidth of the OFDM signal, $\bar{F}^{2}$, defined by

$$
\bar{F}^{2} \doteq \frac{\int_{-\infty}^{\infty}(2 \pi f)^{2} \cdot|X(f)|^{2} d f}{\int_{-\infty}^{\infty}|X(f)|^{2} d f}
$$


can be approximated as follows,

$$
\begin{aligned}
\bar{F}^{2} \simeq \frac{\frac{1}{N_{c}} \sum_{k \in \mathcal{N}_{a}}\left(2 \pi k \cdot F_{s c}\right)^{2} \cdot\left|X\left(k \cdot F_{s c}\right)\right|^{2}}{\frac{1}{N_{c}} \sum_{k \in \mathcal{N}_{a}}\left|X\left(k \cdot F_{s c}\right)\right|^{2}}= \\
=4 \pi^{2} \frac{F_{s c}^{2}}{N_{c}} \sum_{k \in \mathcal{N}_{a}} p_{k}^{2} \cdot k^{2},
\end{aligned}
$$

by considering a rectangular power spectral density (PSD). Thus, disregarding the presence of $\mathrm{CP}$, the CRB for LTE signal pilots, and in general any OFDM signal for one symbol, is

$$
\operatorname{CRB}(\tau)=\frac{T_{s}^{2}}{8 \pi^{2} \cdot \mathrm{SNR} \cdot \sum_{k \in \mathcal{N}_{a}} p_{k}^{2} \cdot k^{2}} .
$$

\section{B. Maximum Likelihood Estimation}

Once the lower bound for timing estimation in LTE has been evaluated, the maximum likelihood estimation (MLE) method is analysed, and preliminary ranging accuracy with LTE pilot signal is presented in additive white Gaussian noise (AWGN) channel. Let us define the received signal $r[n]$ as

$$
r[n]=x\left[n ; n_{\tau}\right]+w[n],
$$

where the discrete time delay (in samples) is $n_{\tau}=\tau \cdot F_{s}$, being $F_{s}$ the sampling frequency, and $w[n]$ the noise component. The MLE method is based on the correlation of the received signal $r[n]$ with a shifted and conjugated version of the reference signal $x[n]$, which is assumed periodical (i.e. circular correlation), in order to find the correlation peak. Thus, the correlation between the received and the transmitted signal is defined by

$$
R_{r x}(\tau) \doteq \sum_{n=0}^{N_{c}-1} r[n] \cdot x_{c}^{*}\left[n+n_{\tau}\right]
$$

where $x_{c}[n]$ is a circular shifted version of the original $x[n]$, resulting in the matched filter of the OFDM signal, whose estimated delay can be expressed as

$$
\hat{\tau}=\frac{T_{s}}{N_{c}} \arg \max _{\tau}\left\{\left|R_{r x}(\tau)\right|^{2}\right\},
$$

where $\tau$ is the time delay in seconds. This method can be efficiently implemented by using the FFT operation, as it is usually done for time and frequency acquisition in GNSS receivers.

\section{Application of Fitz Estimator}

The application of the previous maximum likelihood procedure may pose two main issues. First, its implementation implies a high complexity for a mass-market receiver, and oversampling may even be necessary to avoid quantization errors. Second, this method requires the complete knowledge of the signal, for instance, it cannot be directly applied when data symbols are received. Therefore, a feasible synchronization procedure has to be presented.
Traditionally, in OFDM communications, coarse timing synchronization has been implemented by blind techniques, such as van de Beek algorithm [6], and fine timing synchronization has been achieved with close-loop architectures, such as the well-known delay look loop (DLL) in [7] or [8]. However, open-loop synchronization techniques can also be implemented for fine synchronization purposes, as we show.

The received signal multiplied with the conjugate of the pilot subcarriers in the frequency domain can be expressed, in absence of frequency and phase offsets, as

$$
s[k]=\mathcal{F}\{r[n]\} \cdot d^{*}[k]=e^{j \frac{2 \pi \cdot k \cdot n_{\tau}}{N_{c}}}+w^{\prime}[n],
$$

where $k$ is the subcarrier index and $w^{\prime}[n]$ is the frequency noise contribution. As it can be noticed, we can take advantage of the time shift property of the Fourier transform, $\mathcal{F}\left\{x\left[n \pm n_{\tau}\right]\right\}=X[k] e^{ \pm j \frac{2 \pi \cdot k \cdot n_{\tau}}{N_{c}}}$, to entirely estimate the time delay in the frequency domain. Thus, the TDE estimation becomes a frequency-like estimation problem, and well-known ML frequency estimators can be adopted for time delay estimation after the FFT operation. Examples of low-complexity open-loop carrier frequency estimators are proposed by Kay [9], Fitz [10], Luise and Reggiannini (L\&R) [11], and Xiao et al. [12], and a good reference on the performance and complexity comparison can be found in [13, Sec. 3.2] or [14]. For our application, we choose Fitz estimator, which is able to attain the CRB even at low SNR.

Fitz estimator is an approximation of the ML estimator of the frequency of a sinusoid in white noise, which is the maximum of the periodogram [10]. This practical estimator can be found with the unnormalized autocorrelation function. Nevertheless, the distribution of pilots is scattered over the spectrum, and only few samples of the FFT-output signal can be used. Thus, the application of the Fitz estimator requires a modification on the summation indexes. Let us describe the modified autocorrelation function as

$$
R(m)=\sum_{k \in \mathcal{A}_{0}} s[k] \cdot s^{*}[k-m],
$$

where the subset of available pilot subcarriers $k$ for correlation lag $m$ is expressed as $\mathcal{A}_{0}=\left\{z \in \mathbb{N} \mid z,(z-m) \in \mathcal{N}_{a}\right\}$, which results in $R(m)=e^{j \frac{2 \pi \cdot n_{\tau}}{N_{c}} \cdot m}+w^{\prime \prime}(m)$, being $w^{\prime \prime}(m)$ the noise contribution. Then, the Fitz estimator for time delay estimation of LTE PRS signals can be expressed as

$$
\hat{\tau}=\frac{T_{s}}{2 \pi} \cdot \frac{\sum_{m \in \mathcal{A}_{1}} \arg \{R(m)\}}{\sum_{m \in \mathcal{A}_{1}} m},
$$

where the subset of correlation lags for the PRS pilot distribution is $\mathcal{A}_{1}=\left(\mathcal{A}_{2} \cup \mathcal{A}_{3}\right) \cap \mathcal{A}_{4}$, defined by

$$
\begin{array}{ll}
\mathcal{A}_{2}=\{z \in \mathbb{N} \mid z=6 i & \left.i=1, \ldots, N_{a} / 2-1\right\}, \\
\mathcal{A}_{3}=\{z \in \mathbb{N} \mid z=6 i+1 & \left.i=1, \ldots, N_{a}-1\right\}, \\
\mathcal{A}_{4}=\{z \in \mathbb{N} \mid z \leq M &
\end{array}
$$




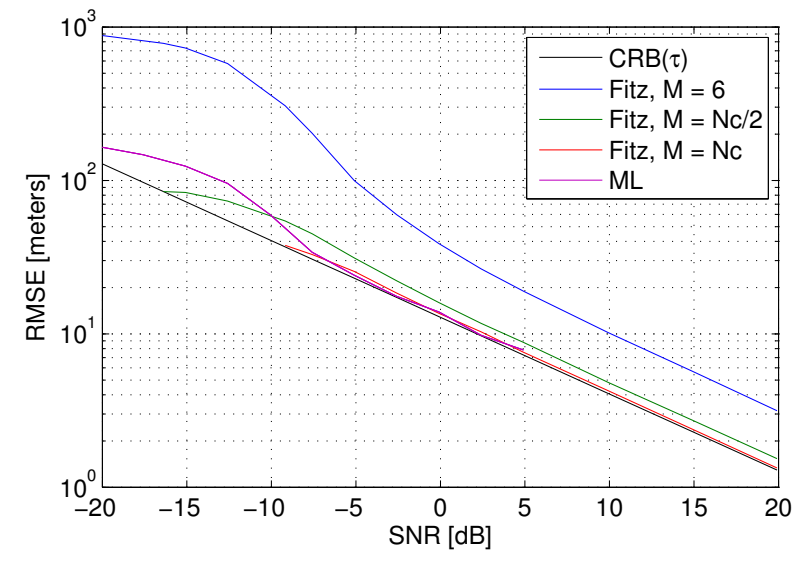

Fig. 2. Comparison between the RMSE of the MLE method and the feasible estimator for the $1.08 \mathrm{MHz}$ PRS signal and only one OFDM symbol.

The value $M$ limits the number of lags, e.g. resulting in

$$
\sum_{m \in \mathcal{A}_{1}} m=\left\{\begin{array}{cl}
6 & \text { if } M=6 \\
\left(3 N_{a}^{2}-5 N_{a}-2\right) / 2 & \text { if } M=N_{c} / 2 \\
N_{a}\left(15 N_{a}-14\right) / 4-1 & \text { if } M=N_{c}
\end{array}\right.
$$

\section{TDE performance assessment}

The timing performance of the maximum likelihood method and the application of Fitz estimator is evaluated by using the root mean square error (RMSE). The RMSE is computed for the LTE pilot signal considering the maximum transmission power according to the bandwidths specified in the standard. In [15, Sec.4.6], the maximum base station (BS) power is specified as follows:

- $43 \mathrm{dBm}$ for bandwidth $\leq 5 \mathrm{MHz}$, and

- $46 \mathrm{dBm}$ for 10,15 and $20 \mathrm{MHz}$ bandwidth.

Assuming the power uniformly distributed among all the active subcarriers, the relative power weight of subcarrier $k$ is

$$
p_{k}=\sqrt{\frac{N_{c}}{N_{a}}}, \quad \text { for } k \in \mathcal{N}_{a} .
$$

For this analysis, pilot signals are transmitted only, thus the power is spread over all the active subcarriers. Using a PRS signal of 12 subcarriers distributed in a bandwidth of $1.08 \mathrm{MHz}$, the RMSE is computed with 10000 Monte-carlo simulations for the maximum likelihood and the application of Fitz estimator. In Fig. 2, the results are compared with the CRB in terms of RMSE in meters, $\operatorname{RMSE}(\hat{\tau})=c \cdot \sqrt{\operatorname{var}(\hat{\tau})}$, being $c$ the speed of light. As it can be noticed, Fitz estimator approximates the ML behaviour and the $\mathrm{CRB}$ when increasing the number of lags $M$. In addition, $M=N_{c} / 2$ is not yet optimal in our application.

\section{CRB FOR OTDOA LOCALIZATION}

The estimation of the user position, $\mathbf{x}=(x, y)^{T}$, can be assessed by means of the CRB for OTDoA localization. For this purpose, the most powerful $K$ base stations with respect to position $\mathbf{x}$ are considered, defining their locations by $\mathbf{x}_{i}=$ $\left(x_{i}, y_{i}\right)^{T}$, where $i=1, \ldots, K$. The range between these base stations and the user is computed with the Euclidean distance of their positions as

$$
d_{i}=\left|\mathbf{x}-\mathbf{x}_{i}\right|=\sqrt{\left(x-x_{i}\right)^{2}+\left(y-y_{i}\right)^{2}} .
$$

The OTDoA localization is based on computing the difference of these range measurements. For this computation, the most powerful base station, which location is $\mathbf{x}_{1}$, is considered the reference BS. Thus, assuming no clock offsets, the LTE network can estimate range differences as

$$
\hat{\mathbf{d}}=\mathbf{d}+\mathbf{n}, \quad \mathbf{n} \sim \mathcal{N}(0, \mathbf{R}),
$$

where $\mathbf{d}$ is defined as the true range differences vector,

$$
\mathbf{d}=\left|\mathbf{x}-\mathbf{x}_{1}\right|-\left|\mathbf{x}-\mathbf{x}_{j}\right|, \quad j=2, \ldots, K,
$$

and $\mathbf{n}$ is the noise vector assumed to be additive white Gaussian noise (AWGN) with constant covariance matrix $\mathbf{R}$,

$$
\mathbf{R}=\left(\begin{array}{cccc}
\sigma_{1}^{2}+\sigma_{2}^{2} & \sigma_{1}^{2} & \cdots & \sigma_{1}^{2} \\
\sigma_{1}^{2} & \sigma_{1}^{2}+\sigma_{3}^{2} & \cdots & \sigma_{1}^{2} \\
\vdots & \vdots & \ddots & \vdots \\
\sigma_{1}^{2} & \sigma_{1}^{2} & \cdots & \sigma_{1}^{2}+\sigma_{K}^{2}
\end{array}\right)
$$

being $\sigma_{i}$ the standard deviation, which is defined by $\operatorname{RMSE}(\hat{\tau})$ for the $\operatorname{CRB}(\tau)$ written in (5). The general derivation of the CRB in AWGN channel can be found in [5, p.47], and it is applied for TDoA in [16], [17]. Although incurring in a penalty, as noted in [17] and [18], the covariance $\mathbf{R}$ is approximated to be constant or non-dependant of the user position, thus the CRB for OTDoA localization results in

$$
\operatorname{CRB}(\mathbf{x})=\left(\mathbf{D}^{T} \mathbf{R}^{-1} \mathbf{D}\right)^{-1},
$$

where

$$
\mathbf{D}=\left(\begin{array}{cc}
\frac{x-x_{1}}{d_{1}}-\frac{x-x_{2}}{d_{2}} & \frac{y-y_{1}}{d_{1}}-\frac{y-y_{2}}{d_{2}} \\
\frac{x-x_{1}}{d_{1}}-\frac{x-x_{3}}{d_{3}} & \frac{y-y_{1}}{d_{1}}-\frac{y-y_{3}}{d_{3}} \\
\vdots & \vdots \\
\frac{x-x_{1}}{d_{1}}-\frac{x-x_{K}}{d_{K}} & \frac{y-y_{1}}{d_{1}}-\frac{x-x_{K}}{d_{K}}
\end{array}\right) .
$$

The position error in meters with respect to the true position $\mathbf{x}$ is finally computed as

$$
\varepsilon_{\mathbf{x}}=\sqrt{\operatorname{tr}(\operatorname{CRB}(\mathbf{x}))} .
$$

\section{Simulation Results in The LTE SCEnARio}

The previous analysis reveals the maximum achievable accuracy in AWGN channel. This analysis can be applied to the LTE scenario by introducing inter-cell interference into the channel model. In [19], system and propagation errors are also considered to assess the LTE positioning performance with a sensitivity analysis. In contrast, our interest is based on the assessment of the LTE localization accuracy from the maximum likelihood point of view. A study in challenging conditions, such as in the presence of severe shadowing or multipath, is left for future work. 


\section{A. Non-coordinated network}

The inter-cell interference is produced due to the single frequency transmission of the different base stations, as is typically planned in cellular networks for spectral efficiency. The received signal from neighbour cells is heavily masked by the strong signal of the serving cell, leading to the so-called near-far effect. Since the network provider decides if data is transmitted during positioning occasions, the PRS pattern can be used inefficiently by interfering the PRS pilots with data of neighbour cells, resulting on a non-coordinated network from the positioning point of view. Our analysis is based on the PRS signal over six resource blocks (RB), i.e. 12 pilot subcarriers along $1.08 \mathrm{MHz}$, and using only one OFDM symbol.

This interference LTE scenario is studied by means of a simulator implemented in MATLAB. The simulation follows the LTE standard [15], and creates a typical cell layout based on a hexagonal grid with three-sectorial base stations (i.e. 3 $\mathrm{dB}$-beamwidth of 65-degree) and inter-site distance of 750 meters. Considering the parameters summarized in Table II, the received signal power from BS $i$ is computed using the expression given in [15, p.14],

$$
P_{r x, i}=P_{t x, i}-\max \left(L_{i}-G_{t x, i}-G_{r x}, \mathrm{MCL}\right),
$$

where $P_{t x, i}$ is the transmitted signal power, $L_{i}$ is the macroscopic pathloss, $G_{t x, i}$ is the transmitter antenna gain, $G_{r x}$ is the receiver antenna gain and MCL is the minimum coupling loss [15], defined as the minimum path loss between mobile and base station antenna connectors. This power budget is used to compute the signal-to-interference plus noise ratio (SINR) in an AWGN channel. The SINR can be expressed as the ratio of signal power to the combined interference and noise power,

$$
\mathrm{SINR}=\frac{P_{r x, i}}{\sum_{j \neq i} P_{r x, j}+N_{r x}},
$$

where $P_{r x, j}$ is the received power from other antenna sectors, which causes the interference, and $N_{r x}$ is the receiver noise floor. As it shown in Fig. 3(a) for BS 1, the SINR drastically decreases near the neighbour base stations, which clearly shows the near-far effect. Applying (23), the position error can be computed for this interference case by using the CRB of (5) with the SINR values and considering five base stations for OTDoA. As it is shown in Fig. 3(b), the errors are only below 100 meters for intermediate areas between base stations, because of their minimal mutual interference, leading to position errors between 20 and 40 meters.

\section{B. Interference cancellation}

In order to reduce the interference impact, the LTE standard already provides inter-cell interference coordination techniques to increase data transmission performance at critical positions of the cell layout, such as at the cell edge. An overview of these techniques can be found in [20], where the interference cancellation (IC) technique can be highlighted. The IC technique is based on reconstructing the signal from the strongest BS and subtracting it from the received signal, in
TABLE II

SPECIFIC SIMULATION PARAMETERS ACCORDING TO [15].

\begin{tabular}{ll}
\hline BS antenna gain & $15 \mathrm{dBi}$ \\
Minimum coupling loss & $70 \mathrm{~dB}$ \\
UE antenna model & Omnidirectional, 0 dBi \\
UE noise figure & $9 \mathrm{~dB}$ \\
Path loss model $^{1}$ & $128.1+37.6 \log _{10}(R) \mathrm{dB}$ \\
\hline
\end{tabular}

${ }^{1} R$ is the propagation distance.

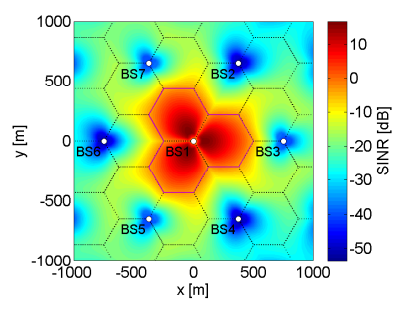

(a) Non-coordinated network: SINR

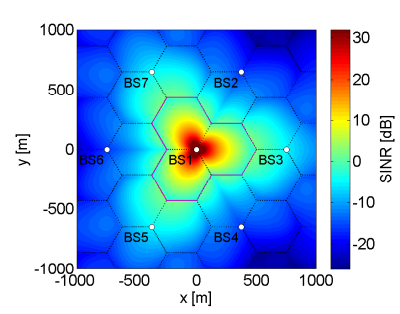

(c) Interference cancellation: SINR

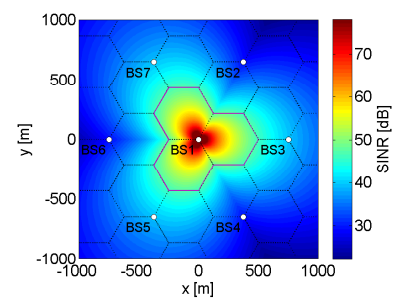

(e) Coordinated network: SINR

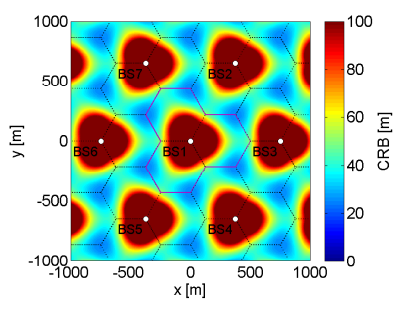

(b) Non-coordinated network: CRB

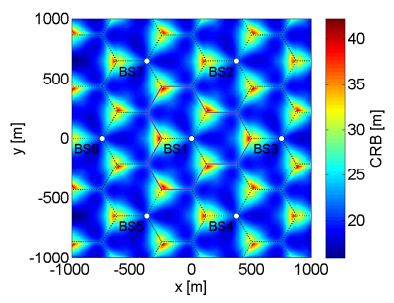

(d) Interference cancellation: CRB

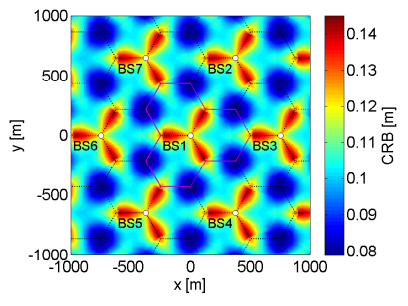

(f) Coordinated network: CRB
Fig. 3. SINR and position error maps computed with the CRB for OTDoA localization for BS 1 transmitting 6 RB of PRS signal.

order to obtain a superposition of the signals from the weaker base stations. Thus, the resulting SINR can be expressed as

$$
\mathrm{SINR}=\frac{P_{r x, i}}{\sum_{\substack{j \neq i \\ j \neq m}} P_{r x, j}+N_{r x}},
$$

where $P_{r x, m}$ is the received power from the strongest BS. This approach is applied for positioning purposes by Mensing et al. in [21], and their results show to enhance the accuracy performance. Nevertheless, errors on the demodulation of the strongest BS signal may deteriorate the cancellation or even increase the interference. This is avoided when the interference source is a pilot signal (i.e. PSS, SSS or CRS signals). Supposing the IC technique is ideal over the PRS signal, the SINR obtained for BS 1, in Fig. 3(c), results on a notable 


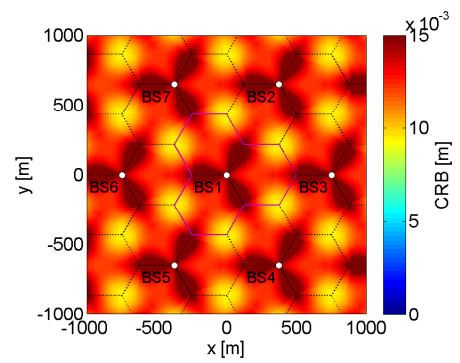

(a) $5 \mathrm{MHz}$ channel bandwidth

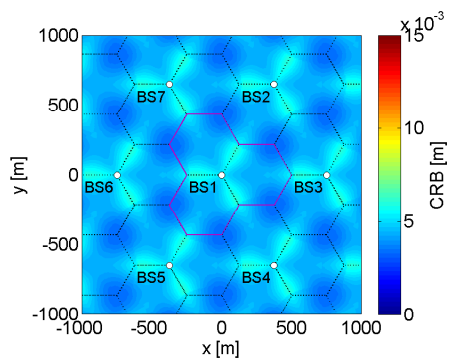

(b) $10 \mathrm{MHz}$ channel bandwidth

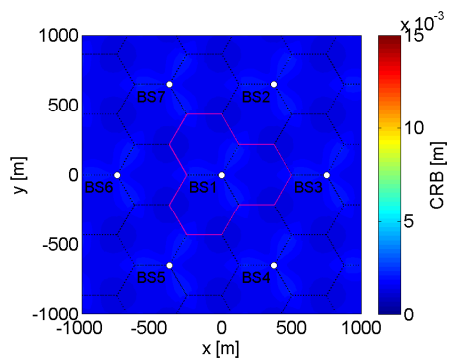

(c) $20 \mathrm{MHz}$ channel bandwidth

Fig. 4. Position errors computed for medium to high PRS bandwidths in a coordinated network.

improvement of the position errors, which are shown in Fig. 3(d) with values between 10 and 40 meters.

\section{Coordinated network}

The interference can be definitely reduced by properly applying the PRS configuration. The PRS capabilities specified in the LTE standard lead to a coordinated network for positioning purposes, by no transmitting data on the PRS bandwidth. Although this coordinated scheme comes at the expense of decreasing the spectral efficiency, the interference avoidance is such that the SINR may be considered equal to the SNR, as is shown in Fig. 3(e), with position errors well below 1 meter, in Fig. 3(f). Sub-centimeter level accuracy can be achieved by spreading the PRS signal up to $20 \mathrm{MHz}$ channel bandwidth (i.e. $100 \mathrm{RB}$ ), as it can be found in Fig. 4. Although the maximum achievable accuracy of positioning reference signals is shown, these results are not realistic because propagation effects, such as shadowing and multipath, are not included, thus considerable lost is expected and future work is required to determine the LTE performance in real environments.

\section{CONCLUSION}

The achievable localization accuracy of the Long Term Evolution (LTE) positioning reference signal (PRS) has been analysed in terms of maximum likelihood estimation, in order to assess its potential as a complimentary system to GNSS. Thus, the Crámer-Rao bound (CRB) for time delay estimation is used as a reference to evaluate the RMSE performance of the ML method (i.e. matched filter) and an adaptation of Fitz estimator, which results practically optimal even at low SNR. Then, the inter-cell interference has been introduced to study the LTE scenario assuming three different cases: noncoordinated network, interference cancellation and coordinated network. Finally, the corresponding position error is computed with the CRB for OTDoA localization. Considering only interference channel, LTE coordinated networks may achieve positioning accuracy below one meter. Future work is necessary to complete the LTE positioning capabilities assessment, for instance, by introducing other propagation effects, such as shadowing or multipath.

\section{ACKNOWLEDGMENT}

This work was supported by the European Space Agency (ESA) under the PRESTIGE programme ESA-P-2010-TEC-
ETN-01 and by the Spanish Government under project TEC2011-28219.

\section{REFERENCES}

[1] 3GPP home page. [Online]. Available: http://www.3GPP.org

[2] J. A. Del Peral-Rosado, J. A. López-Salcedo, G. Seco-Granados, F. Zanier, and M. Crisci, "Preliminary analysis of the positioning capabilities of the positioning reference signal of 3GPP LTE," in Proc. 5th Eur. Workshop on GNSS Signals and Signal Processing, Dec. 2011.

[3] 3GPP TS 36.305, Stage 2 functional specification of User Equipment (UE) positioning in E-UTRAN (Rel. 9), Std.

[4] 3GPP TS 36.211, Physical Channels and Modulation (Rel. 9), Std.

[5] S. Kay, Fundamentals of Statistical Signal Processing: Estimation Theory. Prentice-Hall PTR, 1993-1998.

[6] J. van de Beek, M. Sandell, and P. Borjesson, "ML estimation of time and frequency offset in OFDM systems," IEEE Trans. on Signal Processing, vol. 45, no. 7, pp. 1800-1805, July 1997.

[7] B. Yang, K. Letaief, R. Cheng, and Z. Cao, "An improved combined symbol and sampling clock synchronization method for OFDM systems,' in Proc. WCNC '99, vol. 3, Sept. 1999, pp. 1153-1157.

[8] D. Serant, P. Thevenon, M.-L. Boucheret, O. Julien, C. Macabiau, S. Corazza, M. Dervin, and L. Ries, "Development and validation of an OFDM/DVB-T sensor for positioning," in Proc. IEEE/ION PLANS, May 2010, pp. 988-1001.

[9] S. Kay, "A fast and accurate single frequency estimator," IEEE Trans. on Acoustics, Speech and Signal Processing, vol. 37, no. 12, pp. 19871990, Dec. 1989.

[10] M. Fitz, "Planar filtered techniques for burst mode carrier synchronization," in Proc. IEEE GLOBECOM '91, vol. 1, Dec. 1991, pp. 365 -369.

[11] M. Luise and R. Reggiannini, "Carrier frequency recovery in all-digital modems for burst-mode transmissions," IEEE Trans. on Communications, vol. 43, no. 234, pp. 1169-1178, Feb./Mar./Apr. 1995.

[12] Y.-C. Xiao, P. Wei, X.-C. Xiao, and H.-M. Tai, "Fast and accurate single frequency estimator," Electronics Letters, vol. 40, no. 14, pp. 910-911, July 2004.

[13] U. Mengali and A. D’Andrea, Synchronization Techniques for Digital Receivers. New York: Plenum, 1997.

[14] M. Morelli and U. Mengali, "Feedforward frequency estimation for PSK: A tutorial review," Eur. Trans. on Telecommunications, vol. 9, no. 2, pp. 103-116, Mar./Apr. 1998.

[15] 3GPP TR 36.942, Radio Frequency $(R F)$ system scenarios (Rel. 9), Std.

[16] Y. T. Chan and K. C. Ho, "A simple and efficient estimator for hyperbolic location," IEEE Trans. on Signal Processing, vol. 42, no. 8, pp. 19051915, Aug. 1994.

[17] R. Kaune, J. Horst, and W. Koch, "Accuracy analysis for TDOA localization in sensor networks," in Proc. IEEE FUSION, July 2011.

[18] Y. Wang, G. Leus, and A.-J. van der Veen, "Cramer-Rao bound for range estimation," in Proc. IEEE ICASSP, April 2009, pp. 3301-3304.

[19] R1-092307, "Analysis of UE subframe timing offset measurement sensitivity to OTDoA performance," 3GPP, Alcatel-Lucent, June 2009.

[20] G. Boudreau, J. Panicker, N. Guo, R. Chang, N. Wang, and S. Vrzic, "Interference coordination and cancellation for $4 \mathrm{G}$ networks," IEEE Communications Magazine, vol. 47, no. 4, pp. 74-81, April 2009.

[21] C. Mensing, S. Sand, A. Dammann, and W. Utschick, "Interferenceaware location estimation in cellular OFDM communications systems," in Proc. IEEE ICC '09, June 2009. 